\title{
Dynamic Recrystallization (DRX) as the Mechanism for Sn Whisker Development. Part I: A Model
}

\author{
P.T. VIANCO ${ }^{1,2}$ and J.A. REJENT ${ }^{1}$ \\ 1.-Sandia National Laboratories, Albuquerque, NM, USA. 2.—e-mail: ptvianc@sandia.gov
}

\begin{abstract}
A model is proposed that attributes whisker growth in metals and alloys to dynamic recrystallization (DRX) and, in particular, DRX at the material surface. Each step in the DRX process was correlated to the development of whiskers. The DRX model depends upon the details of the deformation process(es) responsible for new grain initiation and growth. The dependencies exhibited by DRX as a function of deformation strain rate, temperature, and microstructure correlate with the behaviors of whisker development. Anomalous or ultrafast diffusion mechanisms, either by themselves or associated with the deformation structures, provide the means of mass transport necessary to grow whiskers. In Part II of this study, the strain and rate kinetics data are determined for Sn. Parts I and II, together, provide a critical step towards developing a capability to predict the conditions that are likely to cause whisker growth in engineering applications.
\end{abstract}

Key words: Dynamic recrystallization, tin whisker growth

\section{INTRODUCTION}

The topic of tin (Sn) whiskers has captured the attention of the materials engineering community as a result of the increased use of pure Sn surface finishes in the electronics industry. Tin whiskers are not a recent development. Studies in the late 1930 s investigated thin filaments that grew spontaneously from Sn coatings used for the corrosion protection of electronic hardware. It was recognized that these Sn filaments or whiskers could pose a reliability concern by creating short circuits between electrical conductors. The immediate, engineering solution to this phenomenon was to contaminate the Sn coating with $>5$ wt.\% of $\mathrm{Pb}$. These $\mathrm{Sn}-\mathrm{Pb}$ coatings became the requisite finish for high-reliability electronic components.

Today, $\mathrm{Pb}$-containing finishes are being replaced with pure $\mathrm{Sn}$ coatings (defined as $<3$ wt. $\% \mathrm{~Pb}$ ) to meet environmental mandates. Consequentially, reliability concerns have been raised, once again, with respect to Sn whiskers. Current research and development activities have addressed, primarily,

(Received February 23, 2009; accepted June 13, 2009;

published online July 7, 2009) mitigation strategies, which include alternative surface finishes or physical barriers (e.g., conformal coatings) to prevent electrical short circuits. However, there is a need for more in-depth studies that investigate the fundamental mechanism(s) and mass transport rate kinetics underlying Sn whisker development.

The present study was undertaken to develop a theory for whisker development that can be generalized beyond only Sn metal. The proposed underlying process is dynamic recrystallization (DRX). Time-dependent, creep deformation caused by a compressive stress is the mechanism that initiates DRX and then provides the mass transport necessary to sustain the grain growth phase of DRX. It is the latter that is responsible, explicitly, for whisker growth. Establishing DRX as the fundamental process of whisker growth provides an important step towards (a) developing a model of whisker growth and (b) finding a first-principles approach towards mitigating whisker growth.

In this, Part I of the report, a brief review will be made of the DRX phenomenon. Then, a physical model will be proposed that describes whisker growth as a DRX process. Both creep and diffusion data will be examined that provide more quantitative 
benchmarks on the mass transport mechanism. The follow-on, Part II of this report, describes a comprehensive creep study that was performed to document the time-dependent deformation of $\mathrm{Sn}$ under stress and temperature conditions prevalent during whisker development. The test samples were fabricated to have a microstructure that is a scaledup version of that in Sn coatings. The creep strain and steady-state strain rate data established quantitative parameters that were used to bound a DRX-based, whisker growth process.

\section{DYNAMIC RECRYSTALLIZATION}

The attention being given specifically to $\mathrm{Sn}$ whiskers stems largely from their impact on engineering applications. However, whisker formation is a general phenomenon that is not limited to only Sn. Other metals and alloys form whiskers, including $\mathrm{Cd}, \mathrm{In}, \mathrm{Zn}, \mathrm{Au}, \mathrm{Pb}$, and Sn-Pb. ${ }^{1-3}$ Also, whisker development need not necessarily originate only from thin-film coatings. Figure 1a shows a scanning electron microscope (SEM) photograph of whiskers growing from a 52In-48Sn alloy solder joint between $\mathrm{a} \mathrm{Cu}$ pin and Au-coated alumina substrate. The $\mathrm{SEM}$ photograph in Fig. $1 \mathrm{~b}$ shows $\mathrm{Pb}$ whiskers growing out of the $\mathrm{Pb}$-rich phase within a eutectic $\mathrm{Sn}-\mathrm{Pb}$, flip-chip solder ball. The whiskers developed only from the surface of the metallographic crosssection, whiskers were not observed on the original ball surface. A depletion volume around the $\mathrm{Pb}$ whisker, which is not always observed around Sn whiskers, resulted from the limited volume of $\mathrm{Pb}$ available in the individual phase particles. Shorter whiskers have been observed to grow from $\mathrm{Sn}-\mathrm{Pb}$ coatings. Their limited length does not raise concerns with respect to electrical short circuits (Fig. 2). These images, along with the cited Refs. 1-3, indicate that whisker development is a generalized phenomenon of metals and alloys.

It is proposed that DRX is the process responsible for whisker growth. Numerous researchers have considered the possibility that whisker growth results from recrystallization, but not specifically DRX. ${ }^{4-8}$ The DRX process explains many of the general behaviors of the whisker phenomenon as well as observations that are specific to Sn whisker development.

A brief synopsis of DRX is provided. Numerous papers have been published on the topic of DRX, beginning in the $1920 \mathrm{~s}$, because of its importance to the metal forming industry. General treatises on the subject are available in Refs. 9-11. DRX is an enhancement of static recrystallization caused by the simultaneous occurrence of deformation. In contrast, static recrystallization occurs when the strain energy of defect structures is reduced without additional deformation occurring at the same time. The slower the strain rate, the more likely it is for the deformation (strain energy build-up) and recrystallization (strain energy loss) processes to
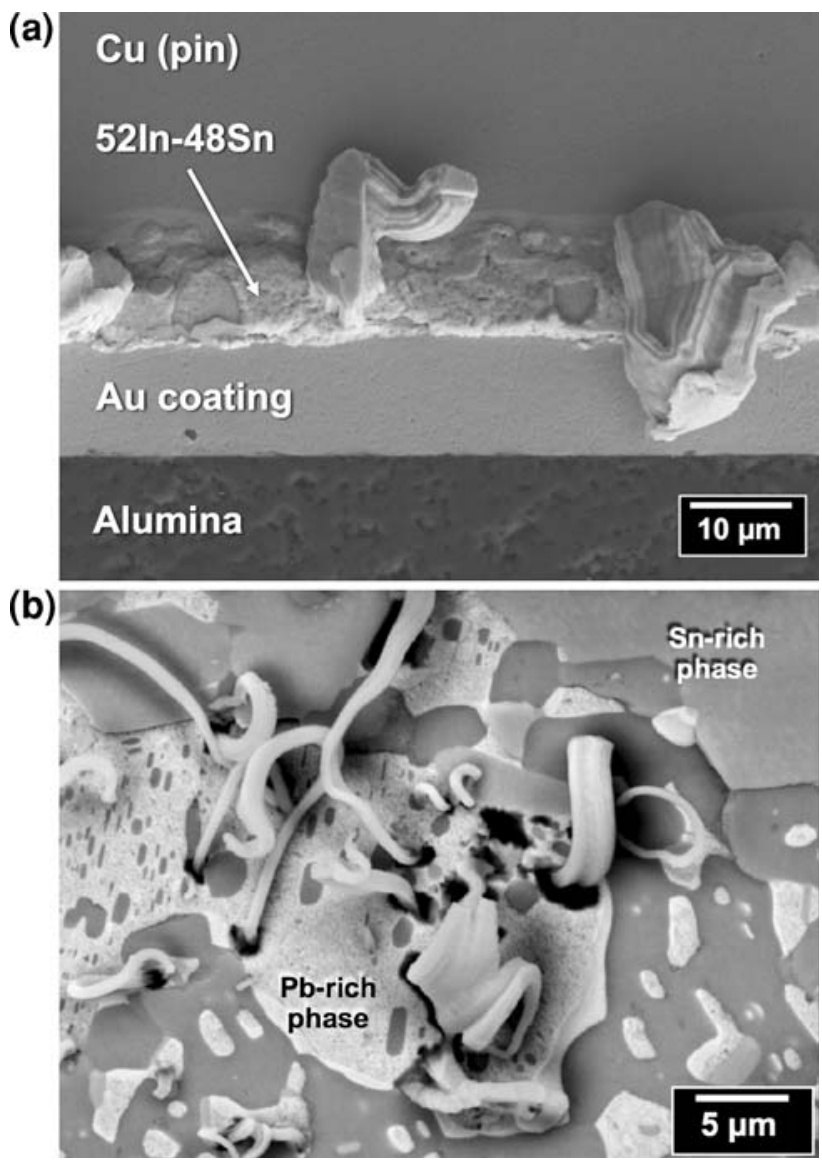

Fig. 1. Scanning electron micrographs showing whisker growth: (a) from a $52 \mathrm{ln}-48 \mathrm{Sn}$ solder joint and (b) from the $\mathrm{Pb}$-rich phase of a eutectic $\mathrm{Sn}-\mathrm{Pb}$ solder, flip-chip solder bump in a metallographic cross-section sample mount.

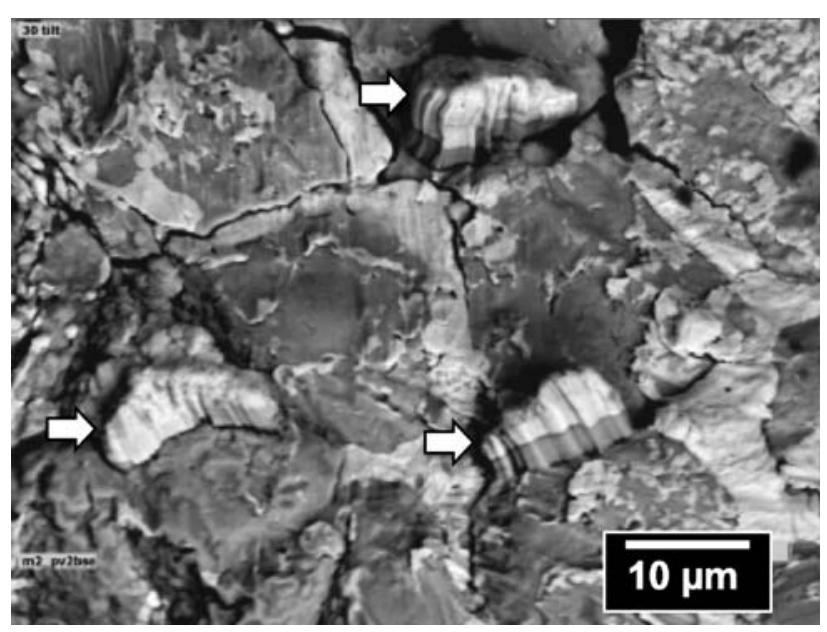

Fig. 2. Scanning electron micrograph of short whiskers growing from a Sn-Pb coating after 1000 thermal cycles $\left(-55^{\circ} \mathrm{C} / 125^{\circ} \mathrm{C} ; 15 \mathrm{~min}\right.$ hold times; $5^{\circ} \mathrm{C} / \mathrm{min}$ ramp rates). The whiskers are often topped by a Pb-rich "cap".

overlap, thereby giving rise to DRX. Mechanistically, the DRX process begins with the build-up of defects-largely dislocation structures-in the 
material as a result of the deformation. The sites of dislocation tangles and pile-ups are typically preexisting grain boundaries. The resulting increase of strain energy provides the added driving force that initiates recrystallization either sooner, or at a lower temperature, than would occur under simply static recrystallization.

Once the strain energy has exceeded a limit, the DRX process proceeds with the nucleation of new grains. These grains are smaller than the original grains; thus, this stage is referred to as grain refinement. These new grains grow under the driving force generated by the annihilation of the dislocation pile-ups and tangles, which removes strain energy from the material. Soon, the growing grains, like the pre-exiting grains, become susceptible to an increased defect density at their boundaries under the applied stress. Dislocations pile-up and tangle at these recently created grain boundaries causing them to become sites of new grain initiation. This cycle continues until the stress has been removed from the material.

It should be noted that there is also the process of dynamic recovery. Static recovery describes the annihilation of point and individual line defects present in the material. Dynamic recovery is an enhancement of the static recovery process because of the creation of additional defects by simultaneous deformation. Recovery processes typically take place at 0.2 to 0.3 of the homologous temperature. In the case of Sn, the corresponding temperature range is $-172^{\circ} \mathrm{C}$ to $-122^{\circ} \mathrm{C}$. Therefore, it is likely that any recovery processes would have taken place well before the occurrence of DRX and thus have little if any impact on Sn whisker development.

There are material properties and deformation conditions that favor DRX. DRX occurs preferably in materials having low stacking fault energies such as Sn. (Dynamic recovery occurs more readily in materials with high stacking fault energy.) Also, DRX is favored under conditions of high temperatures and low strain rates, which allow the grain structure to go through multiple DRX cycles. The resulting stress-strain curves have a cyclic shape after yielding; hence, this condition is referred to as cyclic DRX. For example, iron (Fe) exhibits cyclic DRX when it is deformed at homologous temperatures greater than 0.63 to 0.66 and at strain rates less than $0.002 \mathrm{~s}^{-1} .10$

Conversely, under conditions of low temperatures and very fast strain rates, DRX is more likely to have a single grain refinement event and no appreciable grain growth. This case is referred to as continuous DRX because the accompanying stressstrain curve exhibits a monotonic or continuous, work-hardening behavior.

It is important to note that DRX has been observed during creep deformation of metals and alloys, as well. This case has simply not been studied to the same degree as has the stress-strain behavior because the latter is more applicable to industrial forming processes. Still, the important parameters controlling DRX are temperature and strain rate. Because high temperatures and low strain rates are more apt to occur in creep, the two stages of cyclic DRX-grain refinement and grain growth-are more likely to take place, albeit not always with large amplitudes as is observed in stress-strain tests. Furthermore, it is proposed that whisker growth is the grain growth step of DRX and thus has a greater likelihood to occur under creep conditions.

Because the significant applied parameters for DRX are temperature and the strain rate, the Zener-Hollomon parameter, $Z$, provides a suitable, unified variable to define conditions favorable to DRX. The parameter, $Z$, is expressed as:

$$
Z=\mathrm{d} \varepsilon / \mathrm{d} t[\exp (\Delta H / R T)]
$$

where $\mathrm{d} \varepsilon / \mathrm{d} t$ is the strain rate $\left(\mathrm{s}^{-1}\right) ; \Delta H$ is the apparent activation energy $(\mathrm{kJ} / \mathrm{mol}) ; R$ is the universal gas constant $(8.314 \mathrm{~J} / \mathrm{mol}-\mathrm{K})$; and $T$ is temperature (K). A smaller value of $Z$-low strain rate and higher temperatures-is favorable for cyclic DRX. A slower strain rate does not overwhelm the material with defects, a condition that would favor grain refinement over grain growth. Higher temperatures provide added thermal activation for the grain growth step. Conversely, higher $Z$ values are more conducive to continuous DRX.

Because of the role that deformation has toward the initiation of DRX, in general, a parameter called the critical strain, $\varepsilon_{\mathrm{c}}$, has also been used to predict DRX. Barnett et al. developed the methodology for predicting $\varepsilon_{\mathrm{c}}{ }^{12,13}$ The reader is referred to the cited reference for the detailed derivation. In the case of metals and alloys deformed at homologous temperatures, $T_{\mathrm{h}}$, of 0.5 to 0.7 and subjected to strain rates of $10^{-3} \mathrm{~s}^{-1}$ to $10^{-5} \mathrm{~s}^{-1}$ (which are typical of timeindependent deformation), $\varepsilon_{\mathrm{c}}$ was in the range of 0.1 to 1.0. As reiterated by Sakai and Jones, the critical strain does not provide an indication of whether DRX will be cyclic or continuous, ${ }^{10}$ which is determined by the $Z$ parameter. Similar discussions have addressed the use of a critical stress rather than strain for the initiation of DRX. ${ }^{14}$

An important material parameter of DRX is the grain size. A great deal of study has addressed the grain size effects. ${ }^{9,10,15}$ Those analyses began by first correlating $Z$ with the final DRX grain size, $D_{x}{ }^{9}$ Then, $D_{x}$ was correlated to the initial grain size, $D_{\mathrm{o}}$. Qualitatively, a large $D_{\mathrm{o}}$ encourages grain refinement (new grain initiation) so that stressstrain curves exhibit primarily continuous DRX. More strain is required to (a) generate more defects and then (b) move them across larger grains to pile them up at the boundaries and initiate DRX. Often, there is not enough deformation remaining to repeat the process. On the other hand, a small $D_{\text {o }}$ (per given $Z$ ) is more likely to cause cyclic DRX because the deformation can generate the grain 
growth step after grain refinement. Under the stipulation that whisker growth is the grain growth step of (cyclic) DRX, it is favored by a smaller grain size.

A deformation map can be constructed of $Z$ as a function of $D_{0}$. The map has a critical $Z$ parameter $\left(Z_{\mathrm{c}}\right)$ curve on it. ${ }^{10}$ For a given $D_{\mathrm{o}}$, a value of $Z$ below the corresponding $Z_{\mathrm{c}}$ value (slower $\mathrm{d} \varepsilon / \mathrm{d} t$ or higher $T$ ) is in the cyclic DRX field while a $Z$ value greater than $Z_{\mathrm{c}}$ (faster $\mathrm{d} \varepsilon / \mathrm{d} t$ or lower $T$ ) would result in continuous DRX. For example, the copper $(\mathrm{Cu})$ map shows that a $D_{\text {o }}$ value of $200 \mu \mathrm{m}$ to $300 \mu \mathrm{m}$ corresponds to $Z_{\mathrm{c}}$ of $10^{8} \mathrm{~s}^{-1}$ to $10^{9} \mathrm{~s}^{-1}$. $^{9}$ The development of the $Z$ versus $D_{\mathrm{o}}$ map is based upon determining, empirically or theoretically, the final grain size, $D_{x}$, after a DRX event under the specified $Z$ parameter $(\mathrm{d} \varepsilon / \mathrm{d} t$ and $T)$. The theoretical approach considers $D_{x}$ to be a function of the density of initiation sites. Since initiation sites occur only at the boundaries of the initial grains, the correlation is established between $D_{x}$ and $D_{\mathrm{o}}$. According to either approach, the criterion is generally $D_{\mathrm{o}} \approx 2 D_{x}$. Thus, when $D_{\mathrm{o}}>2 D_{x}$, grain refinement dominates and the DRX is continuous. When $D_{\mathrm{o}}<2 D_{x}$, grain growth is more likely to occur and, as such, cyclic DRX.

Several authors have further broadened the relationship between the critical strain, $\varepsilon_{\mathrm{c}}$, for DRX (again, either cyclic or continuous) and $Z$ that includes the effects of $D_{\mathrm{o}} \cdot{ }^{12,13,16-18}$ That relationship is:

$$
\varepsilon_{\mathrm{c}}=A D_{\mathrm{o}}^{m} Z^{n},
$$

where $A, m$, and $n$ are constants. Although Eq. 2 has not been developed expressly for Sn, calculations of $\varepsilon_{c}$ will be described in Part II that use literature data together with creep strain and strain rate data from those Part II experiments.

Interestingly, subgrain boundaries appear to have a limited direct impact on an active DRX process. ${ }^{17}$ The role of these coherent boundaries is primarily as sources of dislocations during their break-up.

A few studies have examined DRX in Sn. A very comprehensive investigation was conducted by Thijssen. ${ }^{19}$ The author reaffirmed that the limited number of slip systems generally restricts deformation to those grains having the optimum orientation with respect to the applied stress. Cyclic DRX was observed at temperatures of $140^{\circ} \mathrm{C}$ to $190^{\circ} \mathrm{C}$ using compression testing at strain rates of $3.0 \times 10^{-5} \mathrm{~s}^{-1}$ to $7.5 \times 10^{-5} \mathrm{~s}^{-1}$.* Total strains were 0.04 to 0.7 and stresses were $2 \mathrm{MPa}$ to $4 \mathrm{MPa}$. The starting grain size was "small" at $300 \mu \mathrm{m}$ to

\footnotetext{
*Assuming the underlying premise that DRX is responsible for Sn whisker growth, the observation of cyclic DRX within this temperature range implies that $150^{\circ} \mathrm{C}$ annealing treatments are not always an effective mitigation against whiskers. More specific conditions are needed, such as the stipulation that annealing treatments be performed within a specific time interval after deposition.
}

$400 \mu \mathrm{m}$. The grain growth step dominated the metallographic cross-sections, with $D_{x}$ reaching several millimeters. (Clearly, $D_{\mathrm{o}}<2 D_{x}$.) Grain growth was entirely by high-angle grain boundary migration. There were no indications of significant subgrain rotation, which confirms the limited role of subgrain boundaries in the DRX of Sn.

\section{METAL WHISKER GROWTH BY RECRYSTALLIZATION: BACKGROUND}

A long-standing perception of metal whisker growth is that it is more or less a "squeezing the toothpaste tube" phenomenon. Fisher and co-workers pointed out that the finer details of whisker growth discredited such a simple model. ${ }^{4}$ They showed that whisker growth was comprised of an incubation stage followed by a rapid growth step, and then a slower growth stage. They observed that the total whisker length was independent of the applied stress, which directly conflicts with the toothpaste concept. Instead, the stress controlled the growth rate available to reach the maximum length. The authors also recognized that bulk diffusion could not support the rates of mass transport required to grow whiskers; rather, there must be an enhanced diffusion mechanism.

An early work that mentions recrystallization as a source of whisker growth was by Hannay et al. ${ }^{5}$ The authors proposed that whisker growth was preferred, energetically, to more generalized grain growth processes. In fact, recrystallization has a very high driving force. ${ }^{20,21}$ The driving force is nearly four orders of magnitude higher than that of traditional grain growth processes, allowing recrystallization to be the "preferred" process for reducing the strain energy within a material. The studies of Hannay et al. also pointed out the fact that, because $\mathrm{Au}$ whiskers have been observed, surface oxidation was not a controlling factor of whisker growth. A recent study by Moon and co-workers came to a similar conclusion, albeit for Sn-Cu coatings. ${ }^{22}$

Buguslavsky and Bush proposed that abnormal grain growth during recrystallization (static) was responsible for $\mathrm{Sn}$ whisker development. ${ }^{6}$ The authors reiterated the growth process initially described by Lindberg, which was the creation of Frank-Reed loops that glided upward via the associated Frank-Reed dislocation. The loop interiors provided the extra "column" of atoms that created the whisker. An attractive feature of this "FrankReed" process was that it accounted for the singlecrystal columnar (whisker) morphology that was generally observed for metal whiskers. However, a recent study by Courey et al. showed, explicitly, that whiskers are not always single-crystal structures. ${ }^{23}$ This point is confirmed by the SEM photographs in Fig. 3 that show whiskers of $10 \mu \mathrm{m}$ to $25 \mu \mathrm{m}$ diameter growing from an electroplated Sn coating having a grain size of $2 \mu \mathrm{m}$ to $3 \mu \mathrm{m}$. 

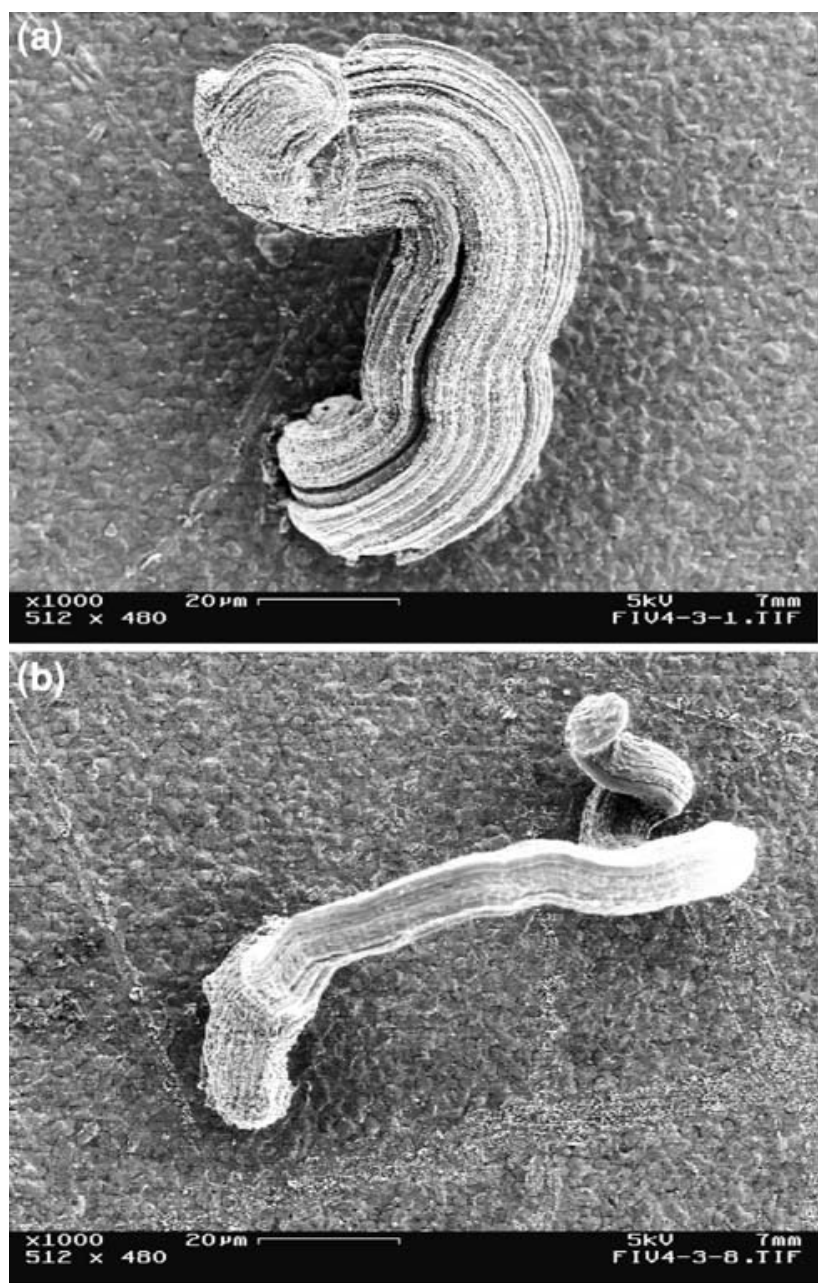

Fig. 3. Scanning electron micrograph of whiskers growing from an electroplated $\mathrm{Sn}$ film having a grain size of $2 \mu \mathrm{m}$ to $3 \mu \mathrm{m}$. The nominal compressive stress was approximately $8 \mathrm{MPa}$; the temperature was $160^{\circ} \mathrm{C}$; time duration was 7 days; and the environment was vacuum.

The striations, and not the whisker diameter, are commensurate with the grain size, implying a coordinated growth process between neighboring grains. Another deficiency of the dislocation loop theory is that it is unlikely that lattice coordination would be able to create a single-crystal loop of $1 \mu \mathrm{m}$ to $5 \mu \mathrm{m}$ in diameter, which is the typical thickness of a metal whisker. ${ }^{24}$

Buguslavsky and Bush described a mass transport mechanism that had the diffusion of Sn atoms and a counterflow of vacancies along pre-existing, high-angle boundaries. They have also suggested that dislocation pipe diffusion may play a role in the mass transport mechanism. It was important to note that Buguslavsky and Bush observed depleted regions next to whiskers, indicating relatively short-range diffusion. On the other hand, Lindburg observed, expressly, the absence of such depleted regions, thereby concluding that long-range diffusion supported whisker development. These two
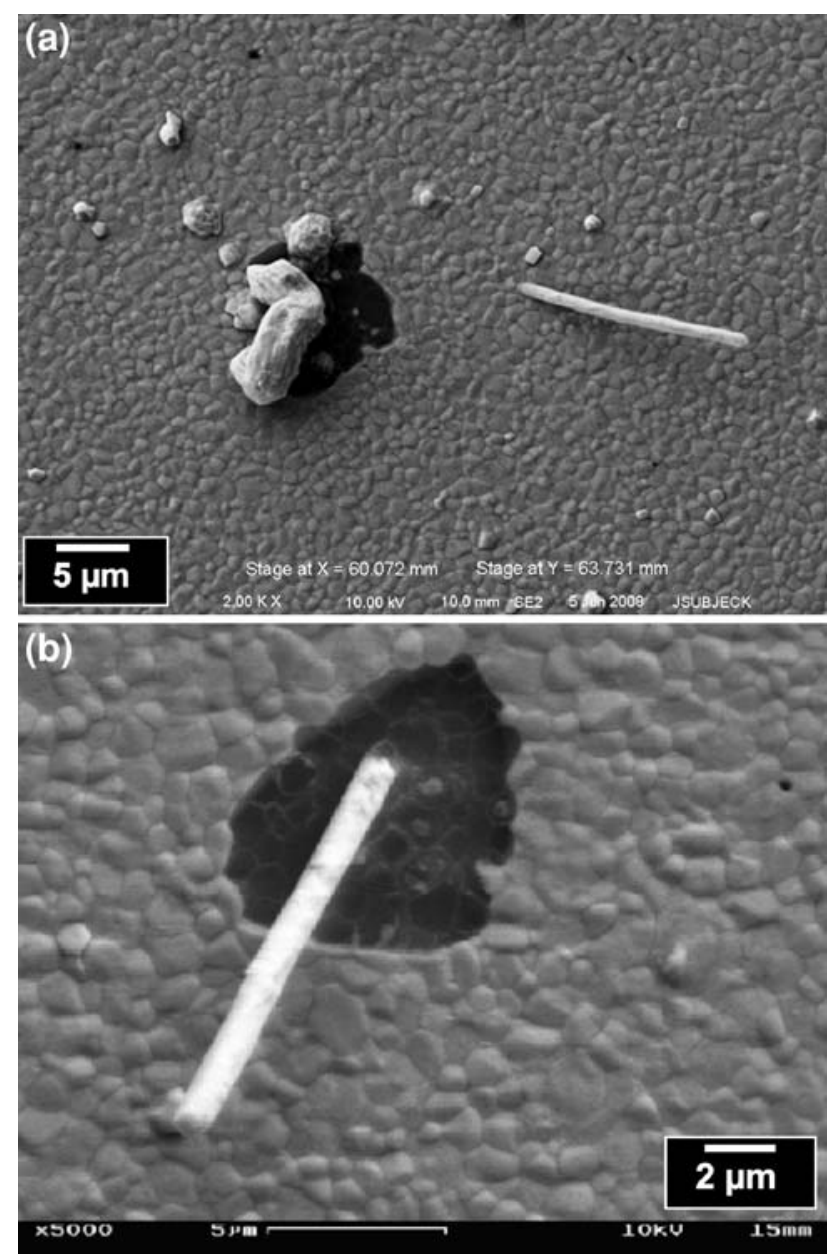

Fig. 4. Scanning electron micrograph of whiskers growing from an evaporated Sn film that was $1 \mu \mathrm{m}$ thick. The nominal compressive stress was approximately $8 \mathrm{MPa}$; the temperature was $160^{\circ} \mathrm{C}$; time duration was 7 days; and the environment was vacuum.

seemingly conflicting observations have persisted throughout studies on whisker growth, including whisker growth from thin evaporated films as shown in Fig. 4. The Sn whiskers grew from a 1- $\mu \mathrm{m}-$ thick evaporated Sn coating that was annealed at $160^{\circ} \mathrm{C}$ for 7 days in vacuum. The compressive stress was approximately $8 \mathrm{MPa}$. Depletion zones were found around some whiskers, but not around others. These different observations suggest that two mass transport mechanisms can generate whisker growth. It has not yet been documented which conditions favor short-range diffusion and those that favor a long-range mechanism.

In summary, previous authors have drawn a correlation between whisker growth and grain growth that takes place during (static) recrystallization. There are several morphological similarities between whiskers and recrystallized grains. The Frank-Reed loop has been proposed as a mechanism to form the observed whisker structure. However, there are also a sufficient number of discrepancies between this mechanism and observed 
whisker properties to preclude the widespread acceptance of static recrystallization and the Frank-Reed loop models for whisker growth.

\section{GENERAL WHISKER GROWTH MODEL BY DYNAMIC RECRYSTALLIZATION}

The present study proposes that whisker growth is a form of DRX. More specifically, whisker growth is a form of DRX that occurs at the surface because metallographic cross-sections have shown that internal grain structures do not exhibit the same elongated geometry as observed in a whisker; rather, they have an equiax geometry. Hillocks reflect DRX grain growth at the surface, but did not form the whisker morphology. ${ }^{25,26}$ In either case, mechanism that underlies DRX must sustain the significant degree of short- and long-range mass transport needed to create these phenomena.

The surface area created by a whisker is relatively large. Tsuji performed an energy balance study of whisker growth. ${ }^{27}$ The author compared the available grain boundary free energy with the surface free energy required to form a whisker and concluded that whisker growth was energetically favorable. Furthermore, Tsuji determined that there is a preference for a whisker to grow out of the surface as opposed to grain growth within the material volume. Thus, this analysis indicates that there is no thermodynamic barrier to whisker formation.

In spite of a large driving force and favorable energy balance, whiskers do not grow spontaneously when the material is exposed only to relatively high homologous temperatures, $T_{\mathrm{h}}(>0.6)$, that is, during static recrystallization. There must be a nominal compressive stress present to sustain the deformation (excess strain energy) needed to support the mass transport necessary for the DRX process to generate whiskers.

The presence of an elevated compressive stress underlies whisker growth. A physical model is that whisker growth is a LeChatelier response to the compressive stress. Material is removed from the specimen volume to reduce the strain energy of the system. The aforementioned "toothpaste tube" extrusion analogy was proposed to describe whisker growth. The specific deformation process would be shear band formation. However, the observed whisker morphology does not lend itself to a shear band process. Aside from morphological differences, whisker growth is observed at stress levels that are well below the yield strength of the metal or alloy. Also, whisker growth is more discriminating as to initiation sites vis-à-vis the grain structure. Lastly, individual whiskers extend to lengths of several tens to hundreds of "grain diameters," which requires a more elaborate mass transport process than the physical displacement of bulk material as in shear band.

In the proposed DRX process, the compressive stress does not explicitly cause whisker growth by the bulk movement of material. Rather, the compressive stress generates inelastic deformation and thus an increase of strain energy that initiates DRX, which is the source of whisker growth from the surface. Recall that DRX can be supported by either time-dependent (creep) or time-independent deformation (stress-strain). The strain rate and temperature, combined together analytically in the $Z$ parameter, are important parameters for DRX.

The proposed DRX mechanism is also compatible with grain size effects on whisker growth. Whiskers, like DRX, are a grain-size-level phenomenon. The initiation of a whisker is the grain refinement step of DRX. The density of whiskers is determined by the number of initiation sites, which is a function of the initial grain size, $D_{\mathrm{o}}$. The smaller the grain size, the greater the number of initiation sites. There would be a large number of initiation sites in coatings and thin films because of their relatively small grain sizes. Once initiation has taken place, the actual lengthening of the whisker is determined by the grain growth step of DRX.

\section{TIN (Sn) WHISKER GROWTH MODEL BY DYNAMIC RECRYSTALLIZATION}

The above discussion drew a correlation between DRX and general whisker growth. That correlation is further refined to Sn whiskers, specifically. Numerous characteristics of Sn whiskers were described by Gaylon ${ }^{28}$ :

The small grain size that favors DRX and the grain growth state is commensurate with the microstructures of thin Sn coatings. Thicker Sn coatings are less susceptible to whiskers because there are more grains to accommodate the stresses, thereby reducing the driving force for DRX.

Elevated temperatures are present. At room temperature, $\mathrm{Sn}$ is at a homologous temperature of 0.59 , which can readily activate DRX.

The internal stresses in most Sn coatings are below the yield stress. ${ }^{29}$ Thus, there is the need for time-dependent (creep) deformation at low strain rates to support DRX for whisker growth. The low strain rates and high homologous temperatures encourage DRX and, more importantly, cyclic DRX with grain growth step. Also, time-dependent deformation would account for the incubation period that was observed by Fisher et al. ${ }^{4}$. The incubation period is the time required to build up the deformation needed to initiate DRX. In a similar manner, whisker formation is significantly delayed with larger grain sizes $(>10 \mu \mathrm{m})$.

Whiskers do not grow everywhere. There are preferred initiation sites. It has been proposed that the few available slip systems in the body-centered tetragonal structure of $\mathrm{Sn}$ is the determining factor for whisker locations. Although creep deformation, and more so, creep that is likely diffusion assisted, is less sensitive to slip-system availability, slip systems that are favorably oriented to the 
compressive stress are required for the generation of dislocations that support the creep process. Of course, smaller grains (e.g., bright Sn finishes) would have a higher probability of such favorably oriented systems and, thus, whisker development.

The DRX model implies that Sn whisker growth is not sensitive to the oxide layer. Early theories on whisker growth from Sn coatings proposed an importance for the oxide layer; however, more recent studies have concluded the contrary. ${ }^{22}$

Annealing treatments have caused inconsistent results in terms of Sn whisker growth. The general premise of an annealing treatment is that it would relieve the compressive stress responsible for whisker development. However, those stresses are relieved via relaxation, which necessarily includes time-dependent deformation that can, in turn, initiate DRX. Because the DRX process is controlled by strain rate, temperature, and grain size, the prevention of whisker growth cannot be achieved by thermal annealing simply on the premise of stress reduction. In fact, these three variables- $\mathrm{d} \varepsilon / \mathrm{d} t, T$, and $D_{\mathrm{o}}$ - can combine to promote whisker growth, or at the very least, have no impact on it, depending upon their values in terms of $Z_{\mathrm{c}}$ and $\varepsilon_{\mathrm{c}}$ discussed earlier. For example, in terms of $D_{\mathrm{o}}$, the Sn grain size changes very little with typical annealing treatments at $150^{\circ} \mathrm{C}$ (e.g., $1 \mathrm{~h}$ to $\left.24 \mathrm{~h}\right) .^{30,31}$ Concurrently, the Sn whisker density did not change significantly as a consequence of the annealing treatment. ${ }^{31}$ A similar observation was made by Cheng et al. on evaporated Sn films $(\sim 1 \mu \mathrm{m}$ grain size $){ }^{32}$
Qualitatively, subjecting the Sn film to an annealing treatment when the compressive stress has peaked stands the greatest likelihood of preventing whiskers per the DRX process. The resulting strain rate is maximized, which favors continuous DRX as opposed to cyclic DRX, the latter having the grain growth step that is directly responsible for whisker growth.

\section{DETAILS OF THE PROPOSED DYNAMIC RECRYSTALLIZATION MODEL FOR WHISKER GROWTH}

The development of a DRX model for general whisker growth requires establishing two processes: (a) the deformation mechanism that initiates DRX and (b) the mass transport mechanism that sustains grain (whisker) growth. Several geometric details discussed below are similar to those described in Ref. 7; however, the mechanism is entirely different. The steps in the proposed model are illustrated in Fig. 5. The compressive stress creates dislocations that pile up at pre-existing grain boundaries (Fig. 5a). The resulting strain energy increases to the point where new grains are initiated as the DRX grain refinement step (Fig. 5b). Note that the whisker does not grow from a pre-existing grain, which is similar to the model proposed by Smetana. As a result, the new grain, and thus whisker grain orientation, need not correlate exactly to the texture of the nearby grains. However, the orientation of the DRX grain may have a second-order dependence
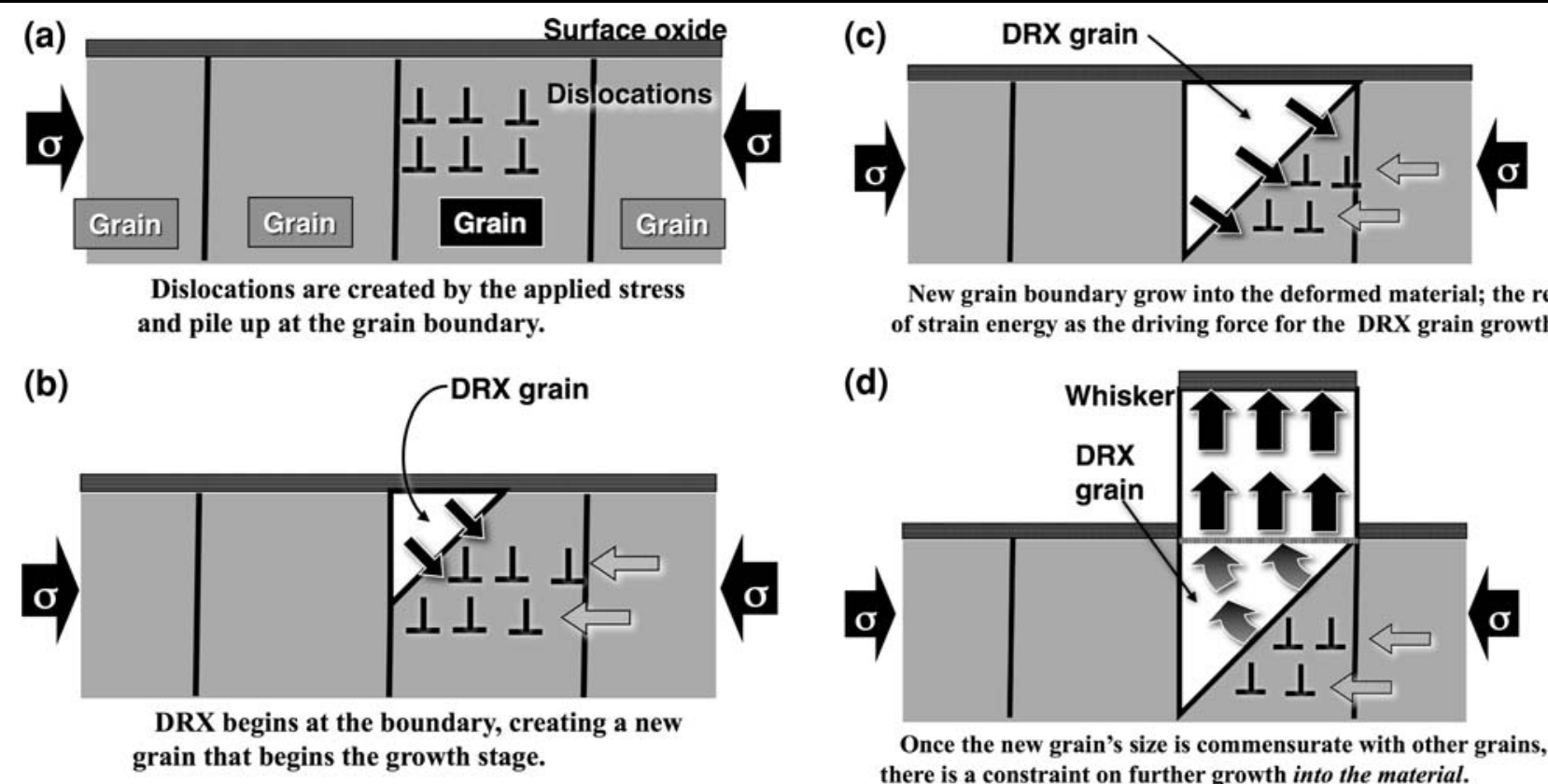

\begin{abstract}
New grain boundary grow into the deformed material; the release of strain energy as the driving force for the DRX grain growth.
\end{abstract}

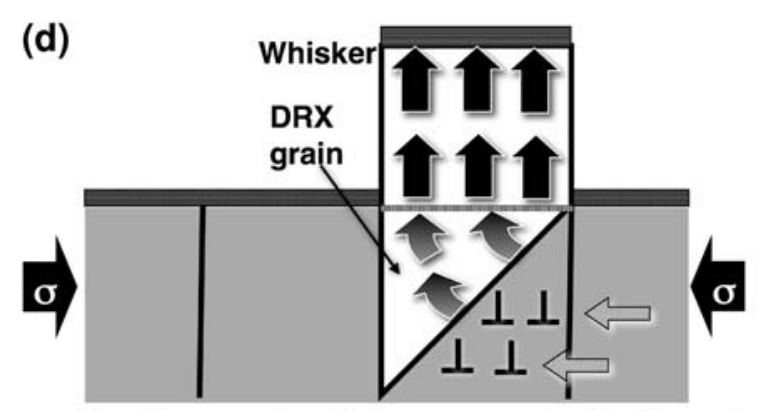

\footnotetext{
Once the new grain's size is commensurate with other grains, there is a constraint on further growth into the material.
}

Fig. 5. Schematic diagram showing the sequence of steps in whisker growth under the proposed DRX model: (a) deformation generates dislocations and other defects that pile up at prior grain boundaries; (b) the strain energy becomes sufficiently high to initiate a new "DRX" grain, which grows into the regions of accumulated defects; (c) the "DRX" grain grows to a size, $D_{x}$, that is similar to the initial grain size $D_{0}$; and (d) there being no further grain growth into deformed material, the grain then grows from the surface to form a whisker as atomic planes are added at the boundary. 
on the texture of neighboring grains because the limited active slip systems in them must be favorably oriented with respect to the compressive stress in order to generate the dislocations. The initial grain size, $D_{\mathrm{o}}$, in the model, which is similar to the film thickness, would support a DRX process for typical Sn coating layers. ${ }^{33,34}$

The minor relationship between the Sn texture and DRX-based whisker growth leads to two observations of Sn whiskers. First, there is the stochastic nature of whisker locations, stress gradients aside. The analogy would be pitting corrosion on metal surfaces. Like the latter analogy, once initiated and the mass transport process is established, whisker growth ensues under the diminishment of the compressive stress and associated system strain energy. Secondly, the new DRX grain and resulting whisker can develop with the most efficient packing structure, which are (001) planes perpendicular to the growth (length) direction. ${ }^{35}$

The newly formed grain grows into the deformed material by boundary migration. This activity is illustrated by the progression shown in Fig. 5b to $5 \mathrm{c}$.

However, the new grain is limited to a size that is commensurate with the existing grain size, $D_{\mathrm{o}}$, as described by several DRX studies (e.g., 9,10), which in the case of coatings is also related to the Sn film thickness. The condition is reached in Fig. 5c. Nevertheless, deformation continues under the compressive stress, and thus so does the driving force to reduce the resulting strain energy. At this point, the whisker growth process begins to take place, as shown in Fig. 5d. In the reference frame of the layer, the boundary between the DRX grain and pre-existing grain remains stationary. However, that boundary moves in a relative sense as the dislocations with their extra atomic half-planes cross the boundary into the reordered DRX grain. Because the DRX grain is constrained from further growth within the layer, it grows from the surface as a whisker. At the left-hand side of the DRX grain/ whisker, grain boundary sliding would occur, which can certainly be supported under the creep conditions. However, as numerous focused ion beam (FIB) images have shown in the literature, the DRX grain growth is likely to grow symmetrically on either side of the original boundary; so, grain boundary sliding is not required for whisker growth.

The proposed mechanism in Fig. 5 comprises the requisite steps for cyclic DRX: deformation to initiate DRX, grain refinement, and lastly grain growth. The more critical step is grain growth, which allows the whisker (grain) to extend to lengths of up to $1000 \mu \mathrm{m}$. The schematic diagram in Fig. 5 suggests that dislocation slip and the provision for the extra half-plane at the boundary is the only mass transport mechanism. However, it is important not to be too restrictive on the mechanism details. Although dislocation slip may be the most physically obvious mass transport mechanism, dislocations can also move by diffusion processes, e.g., climb. Moreover, dislocation structures themselves can provide shortcircuit paths, e.g., pipe diffusion, that can potentially assist the mass transport of Sn atoms.

It is important to appreciate the potential atomic mechanisms that can sustain the mass transport for whisker growth. In particular, numerous authors have investigated so-called "anomalously" or "extremely" fast diffusion processes in metals and alloys. The terms "anomalously," "extremely," and "ultrafast" are used to distinguish these diffusion processes from fast or short-circuit diffusion typically associated with high-angle boundaries (Coble creep). Various mechanisms have been proposed in the studies of this phenomenon, often characterized by very low apparent activation energies $(\Delta H)$. For example, there is the diffusion of $\mathrm{Co}, \mathrm{Au}, \mathrm{Cu}$, and $\mathrm{Pd}$ solutes in $\mathrm{Pb}$ as measured by McLellan et al. ${ }^{36}$ Cited $\Delta H$ values were in the range of $20 \mathrm{~kJ} / \mathrm{mol}$ to $40 \mathrm{~kJ} / \mathrm{mol}$. The authors proposed several possible atomic mechanisms, ranging from single interstitial jumps to coordinated solute atom movements, but with no conclusive evidence for either process. Yeh and Huntington observed extremely fast diffusion of $\mathrm{Ni}$ in single-crystal $\mathrm{Sn}^{37}$ Along the $c$-axis, the apparent activation energy was $18 \mathrm{~kJ} / \mathrm{mol}$, whereas along the $a$-axis the value was $54 \mathrm{~kJ} / \mathrm{mol}$, the latter being more representative of a traditional fast diffusion process. Similar anomalous diffusion has been observed in In by Nakajima and Koiwa. ${ }^{38}$ Vianco et al. observed anomalously fast diffusion of $\mathrm{Pb}$ in Sn, as indicated by the coarsening rate of $\mathrm{Pb}$-rich phase particles in the $\mathrm{Sn}-\mathrm{Pb}$ alloy under isothermal annealing. ${ }^{39}$ The apparent activation energies ranged from $16 \pm 8 \mathrm{~kJ} / \mathrm{mol}$ to $41 \pm 8$ $\mathrm{kJ} / \mathrm{mol}$. Therefore, the published literature indicates that anomalously fast diffusion is possible in elemental Sn.

The anomalously fast diffusion is not always accompanied by a drop in $\Delta H$. Diffusion is also controlled by the pre-exponential jump frequency factor, which can change by several orders of magnitude for interstitial and substitutional mechanisms. ${ }^{40-42}$ The authors of these references agree that anomalously fast diffusion mechanisms can occur along high-density dislocation paths such as dislocation pile-ups, high-angle grain boundaries, and transformation boundaries (twins).

Hwang and Balluffi showed that, when normalized against the melting temperature, the activation energy of grain boundary diffusion decreases with temperature. ${ }^{43}$ This observation suggests that the same trend can be generalized to all diffusion processes involving disordered pathways, whether the latter are grain boundaries or dislocation cores, thus giving rise to a potentially fast mass transport mechanism to support whisker growth.

In summary, there are anomalously fast interstitial diffusion behaviors within metals and alloys that would be very attractive as a mass transport mechanism for whisker growth. Such mechanisms are characterized by low $\Delta H$ values and/or high 
pre-exponential (frequency) coefficients, and can be operative at grain boundaries or through dislocation structures within the grain.

The above instances of anomalously fast diffusion involved interstitial atoms. A study was performed by Boas and Fensham to investigate self-diffusion in single-crystal $\mathrm{Sn}$ using radioactive tracer analysis. ${ }^{44}$ The diffusion rate was nearly two times as fast along the $c$-axis than along the $a$-axis. The $\Delta H$ values were low, being $25 \mathrm{~kJ} / \mathrm{mol}$ along the $a$-axis and $43 \mathrm{~kJ} / \mathrm{mol}$ along the $c$-axis. The authors theorized that the different diffusion rates were caused by dissimilar nearest-neighbor interactions between the two directions. The low $\Delta H$ values indicate that an anomalously fast, self-diffusion mechanism exists in Sn. Furthermore, diffusion rates can be further enhanced by dislocation structures, e.g., by means of dislocation core diffusion, that serve as a contributing factor towards anomalously fast self-diffusion.

As shown in Fig. 5d, whisker growth occurs by the movement of atoms across the DRX grain boundary, proceeding from a relatively disordered state to a more ordered state. If it is assumed that the reordering process across the DRX boundary is similar to that required for grain boundary migration, the latter's rate kinetics would provide a lower bounds for those of whisker growth. It is a lower bound because the added driving force of the strain energy is not fully taken into account. The apparent activation energy for grain boundary migration in $\mathrm{Sn}$ is $33 \mathrm{~kJ} / \mathrm{mol}$ in the temperature range of $152^{\circ} \mathrm{C}$ to $227^{\circ} \mathrm{C} .{ }^{45}$ This value is slightly less than the $40 \mathrm{~kJ} / \mathrm{mol}$ for grain boundary diffusion in $\mathrm{Sn}$ observed by Martin and Perraillon. ${ }^{46}$ This similarity suggests that atomic diffusion across a grain boundary has, at least, a similar $\Delta H$ to atomic diffusion along grain boundaries.

Again, it is worth reiterating that diffusion rates are also dependent upon the pre-exponential jump frequency term. In Martin and Perraillon's study, the value of $\Delta H$ of $40 \mathrm{~kJ} / \mathrm{mol}$ applies to temperatures of $-2^{\circ} \mathrm{C}$ to $160^{\circ} \mathrm{C}$. However, between temperatures of $40^{\circ} \mathrm{C}$ and $160^{\circ} \mathrm{C}$, the pre-exponential term changed by nearly five orders of magnitude.

The present authors gave significant consideration to diffusion-induced grain boundary migration (DIGM) as a possible mechanism for whisker growth. ${ }^{47-49}$ Unlike the traditional DIGM process in which solute atom diffusion drives grain boundary motion, the applied stress would provide the driving force. The mass transport mechanism would have been vacancy diffusion along pre-existing grain boundaries that compensated for Sn atoms used to create the whisker. The DIGM concept would have whiskers grow from pre-existing grains, which is not like the schematic diagram in Fig. 5d. Instead of dislocations, there would be a flow of atoms and vacancies similar to the mechanism proposed in Ref. 7. The apparent activation energy for the grain boundary motion is very similar to that of grain boundary diffusion in light metals. ${ }^{44,45}$ However, DIGM appears to be less viable than DRX because it cannot account for many of the finer details of whisker growth.

It was noted that depletion zones are sometimes observed around a whisker. The circumstances can range from no depletion zone to a zone that extends several grain sizes beyond the whisker root. The presence or absence of the depletion zone indicates a change of mass transport mechanism from a shortrange to a long-range phenomenon, respectively. In the case of a depletion zone, the source of atoms is the neighboring grains, which are rapidly consumed by the DRX driving force to grow the whisker. When diffusion is long range, Sn atoms are supplied from a larger amount of material, thereby reducing the magnitude of depleted mass at any one location. The details, e.g., Sn microstructure, compressive stress or temperature, that lead to the favoring of one mechanism over the other have not been discerned.

\section{ASPECTS OF Sn DEFORMATION PERTINENT TO THE DYNAMIC RECRYSTALLIZATION MODEL}

Whisker development, as a form of DRX (Fig. 5), is a serial process comprising: (a) deformation that raises the strain energy, (b) new grain initiation (refinement), and then (c) grain growth. The grain growth step is responsible, specifically, for whisker growth. The sustained deformation under the compressive stress drives the mass transport mechanism (Fig. 5d). Because deformation is critical to the DRX process and whisker growth, a brief review is made of it in Sn.

The deformation behavior of Sn has been the subject of numerous articles. A thorough review of Sn mechanical properties was provided by Yang and $\mathrm{Li}^{50}$ Studies were cited that described deformation data-primarily creep-under stresses $(0 \mathrm{MPa}$ to $20 \mathrm{MPa})$ and temperatures $\left(0^{\circ} \mathrm{C}\right.$ to $\left.100^{\circ} \mathrm{C}\right)$. These conditions are commensurate with those that generate Sn whiskers. Weertman measured compression creep of single-crystal Sn $\left(8^{\circ}\right.$ off-axis to the $c$-direction). ${ }^{51}$ The apparent activation energy, $\Delta H$, was $50 \mathrm{~kJ} / \mathrm{mol}$ for temperatures less than $120^{\circ} \mathrm{C}$ and stresses of 2.5 $\mathrm{MPa}$ and 6.5 MPa. Later, Weertman and Breen also performed creep studies on singlecrystal Sn of several orientations. ${ }^{52}$ The apparent activation energies were $34 \mathrm{~kJ} / \mathrm{mol}$ to $38 \mathrm{~kJ} / \mathrm{mol}$ along the [110] and [001] directions. These values were less than 0.4 to 0.6 of the $\Delta H$ for bulk diffusion that is usually attributed to grain boundary diffusion processes, implying that even subboundaries had not developed in the samples. ${ }^{53}$ The authors attributed the low values to dislocation core diffusion-assisted climb. Because these experiments were performed on single-crystal Sn, the low activation energies indicate that anomalously fast diffusion processes are possible within the elemental Sn grain. 
Bonar and Craig evaluated the tensile creep of high purity, polycrystalline $\mathrm{Sn}$ in the temperature range of $27^{\circ} \mathrm{C}$ to $77^{\circ} \mathrm{C}$ and a stress of $4.4 \mathrm{MPa} .^{54} \Delta H$ was $40 \mathrm{~kJ} / \mathrm{mol}$. The authors offered no hypothesis as to an active mechanism. Adeva et al. examined the tensile creep properties of polycrystalline $\mathrm{Sn}$ at temperatures of $20^{\circ} \mathrm{C}$ to $190^{\circ} \mathrm{C}$ and stresses of $5 \mathrm{MPa}$ to $8 \mathrm{MPa}{ }^{55}$ Within the limited temperature range of $20^{\circ} \mathrm{C}$ to $80^{\circ} \mathrm{C}$, the apparent activation energy was $35 \mathrm{~kJ} / \mathrm{mol}$ and the power-law stress exponent, $n$, was 6 . The authors attributed the creep to dislocation climb that was controlled by pipe diffusion along the dislocation core. Park et al. examined the impression creep of polycrystalline Sn. ${ }^{56}$ The punch diameter was $100 \mu \mathrm{m}$ and the average grain size was $20 \mu \mathrm{m}$ to $30 \mu \mathrm{m}$. The apparent activation energy was $40 \mathrm{~kJ} / \mathrm{mol}$; the stress exponent was 5 (3 MPa to $20 \mathrm{MPa})$. Those authors also proposed that pipe (core) diffusion controlled the dislocation motion (climb) here.

The results from the mechanical test studies cited above were further analyzed with respect to the deformation mechanism of whisker growth. The stress and temperature regimes were well within those associated with Sn whisker generation. The temperatures represented high homologous values so that diffusion processes had a significant role in the mass transport mechanism and, thus, the latter's rate kinetics. Also, it is likely that there is an anomalously fast diffusion-in this case indicated by a low $\Delta H$ value-supporting creep deformation, which would also be applicable to $\mathrm{Sn}$ whisker development.

Also, it appears that the operative mechanisms that underlie these anomalously fast mass transport behaviors are not necessarily associated with grain boundary diffusion processes since several experiments were performed on single-crystal Sn. Rather, they appear to be a characteristic of the crystallographic structure of the Sn grains, themselves. Thus, if a correlation is drawn between these behaviors and the deformation step in whisker development, then grain boundary diffusion, or any interface diffusion, is not necessarily the active pathway of mass transport.

\section{SUMMARY}

A model has been proposed that attributes the general whisker growth phenomenon in metals and alloys to DRX and, in particular, DRX at the material surface. Each step in the DRX process was correlated to the development of whiskers.

The DRX model is strongly dependent upon the details of the deformation process responsible for grain initiation and growth. The dependencies of deformation on strain rate and temperature conditions as well as the role of material microstructure are compatible between the proposed DRX process and the observed behaviors of whisker development.
Anomalously or ultrafast diffusion remains a viable process for the mass transport mechanism that supports whisker growth through the deformation process.

The concept DIGM was explored, but was considered not to provide a suitable representation of whisker development.

\section{ACKNOWLEDGEMENTS}

The authors wish to thank C. Robino for his thoughtful review of the manuscript and J. Cheng, University of Rochester, NY, for the SEM pictures of the whiskers growing from thin Sn films. Sandia is a multiprogram laboratory operated by Sandia Corporation, a Lockheed Martin Company, for the US Department of Energy's National Nuclear Security Administration under Contract No. DE-AC04-94AL85000.

\section{REFERENCES}

1. H. Leidecker, Other Metal Whiskers (NASA Goddard Space Flight Center, 2008), http://nepp.nasa.gov/whisker/other_ whisker/index_htm.

2. P. Key, Proc. Electronic Components Conference (1975), pp. 155-157.

3. J. Busse, Whiskers of Tin-Lead (Sn-Pb) on Reflowed Die Attach Solder Used in the Manufacture of a Laser Diode Array (NASA Goddard Space Flight Center, 2003), http://nepp.nasa.gov/whisker/other_whisker/index_htm.

4. R. Fisher, L. Darken, and K. Carroll, Acta Met. 2, 368 (1954).

5. N. Hannay, W. Kaiser, and C. Thurmond, Annu. Rev. Phys. Chem. 11, 407 (1960).

6. I. Boguslavsky and P. Bush, Proc. APEX 2003 (2003), pp. S12-4-1-S12-4-10.

7. J. Smetana, Presentation, iNEMI Tin Whisker Workshop during the Electronic Components and Technology Conference (2005).

8. U. Lindberg, Acta Met. 24, 181 (1976).

9. V.G. Garcia Ferendez (Ph.D. Thesis, Escola Tecnica Superior d'Enginyeria Industrial de Barcelona, U. Politecnica de Catalunya, Bacelona, Spain, 2004).

10. T. Sakai and J. Jones, Acta Met. 32, 189 (1984).

11. H. McQueen, Mater. Sci. Eng. A 387-389, 203 (2004).

12. M. Barnett, G. Kelly, and P. Hodgson, Scr. Mater. 43, 365 (2000).

13. M. Barnett, G. Kelly, and P. Hodgson, Metall. Mater. Trans. A 33A, 1893 (2002).

14. A. Najafizadeh and J. Jonas, ISIJ Inter. 46, 1679 (2006).

15. F. Montheillet and J.-P. Thomas, Metallic Materials with High Structural Efficiency (Netherlands: Kluwer Academic, 2004), pp. 357-368.

16. S. Serajzadeh, Model. Simul. Mater. Sci. Eng. 12, 1185 (2004).

17. L. Dougherty, I. Robertson, and J. Vetrano, Acta Mater. 51, 4367 (2003).

18. I. Salvatori, T. Inoue, and K. Nagai, ISIJ Int. 42, 744 (2002).

19. F. Thijssen, Effect of Strain on Microstructural Evolution During Dynamic Recrystallization: Experiments on Tin (Ph.D. Thesis, Ultrecht University, Netherlands, 2004).

20. D. Smith, C. Rae, and C. Grovenor, Grain Boundary Structure and Kinetics (Materials Park, OH: ASM, Intl., 1979), pp. 337-371.

21. F. Haessner and S. Hoffman, Recrystallization of Metallic Materials (Stuttgart, Germany: Dr. Riederer Verlag GmbH, 1978), pp. 63-95.

22. K.-W. Moon, C. Johnson, M. Williams, O. Kongstein, G. Strafford, C. Handwerker, and W. Boettinger, J. Electron. Mater. 34, L31 (2005). 
23. K. Courey, S. Asfour, J. Bayliss, L. Ludwig, and M. Zapata, IEEE Trans. Electron. Packag. Manuf. 31, 32 (2008).

24. G. Gaylon, iNEMI Monograph (Herdon, VA: iNEMI, 2003).

25. M. Williams, K.-W. Moon, W. Boettinger, D. Josell, and A. Deal, J. Electron. Mater. 36, 214 (2007).

26. J. Cheng, S. Chen, P. Vianco, and J. Li, Proc. 58th Electronic Components and Technology Conference (2008) CD-ROM.

27. K. Tsuji, Proc. IPC/JEDEC Conference (2003), pp. 169-186.

28. G. Gaylon, IEEE Trans. EPM 28, 94 (2005).

29. W. Choi, Y. Lee, K. Tu, N. Tamura, R. Celestre, A. MacDowell, Y. Bong, L. Nguyen, and G. Shen, Proc. 52nd Electronic Components and Technology Conference (2002), pp. 628-633

30. P. Vianco, J. Rejent, and A. Kilgo, J. Electron. Mater. 33, 1473 (2004)

31. Y. Fukuda (PhD. Thesis, University of Maryland, College Park, MD, 2005).

32. J. Cheng (University of Rochester, 2008), unpublished data.

33. C. Sellars, Philos. Trans. R. Soc. Lond. A 288, 147 (1978).

34. M. Wahabi, L. Gavard, F. Montheillet, J. Cabrera, and J. Prado, Acta Mater. 53, 4605 (2005).

35. W. Choi, G. Galyon, K. Tu, and T. Lee, Handbook of LeadFree Solder Technology for Microelectronic Applications, ed. K. Puttlitz and K. Stalter (New York, NY: MarcelDekker, 2004), pp. 851-913.

36. R. McLellan, C. Ko, and F. Brotzen, Acta Metall. Mater. 38, 2161 (1990).

37. D. Yeh and H. Huntington, Phys. Rev. Lett. 53, 1469 (1984).

38. H. Nakajima and M. Koiwa, ISIJ Int. 31, 757 (1991).
39. P. Vianco, J. Rejent, G. Zender, and A. Kilgo, J. Mater. Res. 20, 1563 (2005).

40. C. Ning and Y. Zongsen, J. Mater. Sci. Lett. 14, 557 (1995).

41. Y. Kamon, H. Harima, A. Yanase, and H. KatayamaYoshida, Physica B 308-310, 391 (2001).

42. G. Vogl, W. Mickeley, A. Heidemann, and W. Petry, Phys. Rev. Lett. 53, 934 (1984).

43. J. Hwang and R. Balluffi, Scr. Met. 12, 709 (1978).

44. W. Boas and P. Fensham, Nature 164, 1127 (1949).

45. F. Haessner, ed., Recrystallization of Metallic Materials (Stuttgart, Germany: Riederer Verlag GmbH, 1978), p. 67.

46. G. Martin and B. Perraillon, Grain Boundary Structure and Kinetics (Materials Park, OH: ASM, Inter., 1980), p. 280.

47. B. Gupta, M. Madhuri, and S. Gupta, Acta Mater. 51, 4991 (2003).

48. C. Ma and W. Gust, Scr. Met. 30, 509 (1994).

49. F. Den Broeder, M. Klerk, J. Vandenberg, and R. Hamm, Acta Met. 31, 285 (1983).

50. F. Yang and J. Li, J. Mater. Sci. 18, 191 (2007).

51. J. Weertman, J. Appl. Phys. 28, 196 (1955).

52. J. Breen and J. Weertman, J. Met. 72, 1230 (1955).

53. P. Shewmon, Diffusion in Solids, 2nd ed. (Warrendale, PA: TMS, 1989), pp. 189-199.

54. L. Bonar and G. Craig, Can. J. Phys. 36, 1445 (1958).

55. P. Adeva, G. Caruana, O. Ruano, and M. Torralba, Mater. Sci. Eng. A 194, 17 (1995).

56. C. Park, X. Long, S. Haberman, S. Ma, I. Dutta, R. Mahajan, and S. Jadhav, J. Mater. Sci. 42, 5182 (2007). 